\title{
Comparative Transcriptome Analysis of the Gills from the Chinese Mitten Crab (Eriocheir japonica sinensis) Exposed to the Heavy Metal Cadmium
}

\author{
Dan Tang ${ }^{1}$, Huayun Guo ${ }^{1}$, Xueling Shi ${ }^{1}$, Zhengfei Wang ${ }^{1, *}$ (I) \\ ${ }^{1}$ Yancheng Teachers University, School of Ocean and Biological Engineering, Yancheng, China
}

\section{Article History}

Received 20 November 2018

Accepted 27 November 2019

First Online 04 December 2019

\section{Corresponding Author}

Tel.: 86-515-88233587

E-mail:wangzf@yctu.edu.cn

\section{Keywords}

Transcriptome

Chinese mitten crab

Gill

Cadmium stress

Detoxification

\begin{abstract}
Heavy metal Cadmium (Cd) pollution has become a potential environmental problem, and it threatens aquatic organisms including economic crabs and shrimps. Chinese mitten crab is a native species in East Asia, and it is the most important economic crab species in China. However, there are only a few studies about gills responding to $\mathrm{Cd}$ stress in the Chinese mitten crab at a molecular level. To gain better understanding of the oxidative stress system (antioxidant defense) of the $E$. j. sinensis, in this study, RNASeq was applied to obtain gene expression profile from the mitten crab's gills in response to $\mathrm{Cd}$ for $24 \mathrm{~h}$, and yielding $39,612,948$ high-quality clean reads from which 570,847 transcripts and 504,889 unigenes are assembled by removing redundancy and transcripts with low coverage. A total of 1544 differential expression genes (DGEs) are identified. GO functional analysis and KEGG pathway analysis indicated some vital pathways and genes in response to the challenge. Four genes related to immune response and detoxification are selected to validate the DGE results by qRT-PCR. This comparative transcriptome provides valuable molecular information about on the mechanisms of the Chinese mitten crab responding to $\mathrm{Cd}$ that lays the foundation for further understanding of heavy metals stress.
\end{abstract}

\section{Introduction}

The Chinese mitten crab (Eriocheir japonica sinensis) is a widely-distributed group in streams and rivers along the eastern pacific coast of China to the Korean Peninsula (Wang et al., 2018; Xiong et al., 2001). The Chinese mitten crab has abundant nutrition and delicate taste, making it becomes a common food today and has high market demand and price in China (Li et al., 2018; Wu et al., 2007). The annual production of $E$. $j$. sinensis has increased sharply during the past two decades which is approximately 812,103 tons in 2016 (China Fisheries Yearbook, 2017). Thus, E. j. sinensis has become one of the most prevalent and commercially important aquaculture crabs in China. In addition, E. $j$. sinensis always was considered as a model organism in aquaculture studies (Cui et al., 2013).
Over the past few decades, with the accelerating industrialization and anthropogenic activities, more and more waters have been polluted by high levels of heavy metals (Wang et al., 2015). As a consequence, heavy metal pollution has become a serious environmental problem, producing deleterious impacts on the ecosystem. Particularly, it threatens aquatic organisms including economic crabs and shrimps (Fu and Qi 2011). As one of the most hazardous heavy metals, Cadmium (Cd) is a non-essential and potentially toxic metal because it cannot be degraded in bioaccumulation and can be accumulated in humans via food chain (Gaspic et al., 2002), which would may result in morphological deformities, physiological dysfunctions and even death (Sun et al., 2016). Cd can cause the production and accumulation of reactive oxygen species (ROS) which is known to induce oxidization of biological 
macromolecules and variously physiological damage to animal tissues and organs (Thévenod 2009). Furthermore, Cd also generates DNA strand breaks and gene inactivation (Manna and Sil 2009), and alters gene expression to regulate activities of various enzymes (Overstreet 2000). Previous studies showed that effects of $\mathrm{Cd}$ exposure in many crustaceans which including Sinopotamon henanense (Sun et al., 2016), Macrobrachium rosenbergii (HAKaoud and ARezk 2011), and Gammarus fossarum (Silvestre et al., 2006). However, up to date, the research about $E$. j. sinensis is limited on the knowledge about the molecular basis of responding to $\mathrm{Cd}$ stress.

In crustaceans, the gills are multifunctional and generally regarded as a major organ of respiration (Burnett and Mcmahon 1985) and osmoregulation ( $\mathrm{Hu}$ et al., 2015; Weihrauch et al., 2001). Additionally, the gills also play a critical role in xenobiotics entering organisms, material absorption and biological transformation because it associates with ambient environment directly (Wang et al., 2013). Previous studies have reported $E$. j. sinensis are threatened by $\mathrm{Cd}$, but mostly focused on tissue-specific cadmium accumulation (Silvestre et al., 2005; Wang et al., 2001). Silvestre et al., (2005) found that the anterior gill in $E$. j. sinensis has highest level of cadmium accumulation, followed by the hepatopancreas and posterior gill. However, the studies on the molecular responses of differentially expressed genes are scarce in Chinese mitten crab gills exposed to $\mathrm{Cd}$.

In recent years, with the development of nextgeneration sequencing, de novo transcriptomic approaches have been increasingly used to investigate physiological process and molecular characteristics of model and non-model organisms (Lou et al., 2017). Additionally, de novo transcriptome provides great breadth and depth of information that can be used to catalog all the genes expressed in the tissue, and facilitates detailed functional research of various proteins (Bain et al., 2016). To get better understanding about the molecular basis responses to $C d$ stress in $E$. $j$. sinensis, we used the comparative transcriptome of the gills to analyze the differentially expressed genes (DEGs) between exposed and unexposed to $\mathrm{Cd}$ by using Illumina sequencing platform and quantitative real-time PCR (RTqPCR). The goal of this study is to improve a better understanding of molecular responses of $E$. $j$. sinensis when exposed to $\mathrm{Cd}$ and to provide a theoretical basis for the further researches on heavy metals stress.

\section{Materials and Methods}

\section{Animals and Exposures}

E. j. sinensis were purchased from Yangguang market in Yancheng, Jiangsu Province, China, on October 18th, 2017. Before experiments, the crabs were acclimated 2 days. Crabs were randomly divided to the treated group and the control group. The treated group of three crabs was exposed to $\mathrm{Cd}$ for 24 hours at a concentration of $1 \mathrm{mg} / \mathrm{L}$ prepared by dissolving $2.036 \mathrm{mg}$ of $\mathrm{CdCl}_{2} \cdot 2.5 \mathrm{H}_{2} \mathrm{O}$ in $1 \mathrm{~L}$ of clean water (without $\mathrm{Cd}$ ). The control group of three crabs was maintained under clean water for 24 hours at $20 \pm 0.5^{\circ} \mathrm{C}$. The crabs of each group were settled at tanks $(36 \mathrm{~cm} \times 20 \mathrm{~cm} \times 18 \mathrm{~cm})$ which contain one third water. During the whole processes, crabs were not fed. Then the gills samples of the $E$. j. sinensis from each group were rapidly removed, snap-frozen in liquid nitrogen, placed into $1.5 \mathrm{ml}$ RNasefree tubes and immediately stored at $-80^{\circ} \mathrm{C}$ prior to the RNA extraction, respectively.

\section{RNA Extraction, cDNA Library Preparation and Illumina Sequencing}

Total RNA was isolated from the composite sample using a standard TRIzol Reagent Kit according to the manufacturer's instructions. RNA purity was confirmed spectrophotometrically using a NanoDrop 2000 and integrity were assessed using an Agilent 2100 Bioanalyser (Agilent Technologies, USA). Briefly, mRNA was purified using oligo (dT) magnetic beads. Purified mRNA was fragmented into smaller fragments (about $200 \mathrm{bp}$ ) by using a fragmentation buffer. Then, randomhexamer primers and reverse transcriptase were used to synthesize the first-strand. Next, Second-strand cDNA synthesis was subsequently performed as a stable double-stranded structure, after which end repair and addition of a base at $3^{\prime}$ ends were conducted. The suitable fragments were purified by $2 \%$ agarose gel electrophoresis and enriched by PCR amplification for 15 cycles to create a cDNA library. Finally, the library was conducted by Illumina HiSeq ${ }^{\mathrm{TM}} 2000$ platform (Illumina Inc.).

\section{Assembly and Annotation}

In this study, the SeqPrep
(https://github.com/jstjohn/SeqPrep) and Sickle (https://github.com/najoshi/sickles) software were used to filter raw reads in FASTQ format by removing reads with sequencing adaptors, low quality (quality scores $\leq 20$ ) and unknown nucleotides ( $\mathrm{N}$ ratio $>10 \%$ ). Following quality filtering, all downstream analyses were based on clean, high-quality data, and then clean reads were de novo assembled using Trinity software package (Haas et al., 2013). Longest transcripts of each gene were pooled as "unigenes".

To search for the likely homologues of proteins with known function, all the unigenes matched with sequences in the BLAST against the Non-redundant (NR) database, String, Swiss-Prot protein database, Protein family database (Pfam), Kyoto Encyclopedia of Genes and Genome (KEGG) classification, Clusters of Orthologous Groups (COG) of proteins and Gene ontology (GO) databases, with a cut-off E-value of 1e-5 (Altschul et al., 1997). GO databases were mapped to 
sequences based on their alignment using the Blast2GO software (Delanghe 2005).

\section{Identification of Differentially Expressed Unigenes}

To identify differential expression genes (DEGs) between two samples, the expression level of each unigene was measured by using the Fragments Per Kilobase of exon model per Million mapped reads (FPKM) method (Mortazavi et al., 2008). Differential expression analysis between samples was performed using edgeR (http://www.bioconductor.org/packages/2.12/bioc/ht ml/edgeR). Genes were considered significantly differentially expressed (DEGs) when FDR (false discovery rate) $<0.05$ and $\log _{2}|F C|>=1$. GO term enrichment analysis and KEGG pathway functional enrichment analysis of the differentially expressed genes to determine the DEG functions and biological pathways by Goatools (https://github.com/tanghaibao/GOatools) and KOBAS (http://kobas.cbi.pku.edu.cn/home.do), respectively. We defined the corrected $p$ value $<=0.05$ as significantly enriched (Kanehisa et al., 2008).

\section{Expression Analysis by Real-Time Quantitative PCR (qRT-PCR)}

A total of 4 up-regulated genes associated with $\mathrm{Cd}$ stress were selected for qRT-PCR analysis to validate the Illumina sequencing result and the expression pattern. The gene-specific primers were designed by Primer Premier 5.0 (Table 1). Total RNA was extracted from the gills of three crabs in each group using TRIzol (Invitrogen). qRT-PCR analysis was performed on an Applied Biosystem 7500 real-time PCR system (Applied Biosystems, Thermo Fisher Scientific, Waltham, MA, USA) with $2 \times$ SYBR Green qPCR Mix (Aidlab Biotechnologies Co., Ltd., China). The qRT-PCR reaction system $(25 \mu \mathrm{l})$ consisted of $12.5 \mu \mathrm{L}$ of $2 \times$ SYBR qPCR Mix, $1 \mu \mathrm{L}$ of forward and reverse primers, $1 \mu \mathrm{L}$ of cDNA, and $10.5 \mu \mathrm{L}$ of RNase-free $\mathrm{H}_{2} \mathrm{O}$. $\beta$-actin was used as the reference gene to normalize target gene expression. The PCR program was set with an incubation step: $3 \mathrm{~min}$ at $95^{\circ} \mathrm{C}, 40$ cycles of $15 \mathrm{~s}$ at $94^{\circ} \mathrm{C}, 15 \mathrm{~s}$ at $55^{\circ} \mathrm{C}$ and $25 \mathrm{~s}$ at $72{ }^{\circ} \mathrm{C}$, followed with the standard dissociation cycle. Each gene was repeated three technique replications and $2^{-\triangle \Delta C T}$ method was used for analyzing relative quantification (Thomas et al., 2008).

\section{Results and Discussion}

\section{Illumina Sequencing and De Novo Assembly}

To get an overview of the transcriptomic characteristics of the Cd stress, two cDNA libraries were constructed between the gills of exposed and unexposed $E$. j. sinensis. We obtained 39,612,948 total raw reads and $34,265,370$ total raw reads from treated group and control group samples, respectively. The raw reads were further filtered into clean reads. After assembly, we obtained 570,847 transcripts, which were further clustered into 504,889 unigenes. The unigenes comprised $188,995,620$ bases, and the N50 length of $364 \mathrm{bp}$ with an average GC content of approximately $44.11 \%$. The average length, largest length and smallest length were 374.33bp, 50681bp and 201bp, respectively. Detailed information relevant to overall assembly quality was summarized in Table 2.

\section{Functional Annotation and Classification}

All the unigenes were aligned to NR, String, SwissProt, Pfam, GO and KEGG database, with a cut-off Evalue of $1 \mathrm{e}-5$. A total of 71,968 unigenes (13.11\% of all unigenes) had significant hits in at least one of these databases, including 60,587 (84.19\%), 47,038 (65.36\%), $42,963(59.70 \%)$ and 42,357 (58.86\%) were mapped to String, NR, Swiss-Prot and GO databases, respectively. However, comparing to four databases, only 25738 (35.76\%) and 24773 (34.42\%) of the total sequences were matched against Pfam and KEGG databases. These annotation and classification will facilitate the following interpretation of the gene function in $E$. $j$. sinensis.

From the annotated information of six databases, all unigenes were annotated through Blast against the NCBI non-redundant (nr) protein database built for the invertebrate. The Blast top-hit species distribution of the annotated unigenes showed the highest homology to the Hyalella azteca (9838, 20.92\%), followed by Zootermopsis nevadensis (1547, 3.29\%), Trichuris trichiura (1121, 2.38\%), Capitella teleta (993, 2.11\%) and Plutella xylostella (950, 2.02\%). Except for the top 20 species with sequence hits, the largest number of the unigenes was hits in the other species (23619, 50.21\%) due to limited genome information in curstaceans (Figure 1).

Table 1. Real-time quantitative PCR primers used in this study

\begin{tabular}{lcc}
\hline Gene Name & Forward primer sequence $\left(5^{\prime}-3^{\prime}\right)$ & Reverse primer sequence $\left(5^{\prime}-3^{\prime}\right)$ \\
\hline B-actin & GATGGTGGGAATGGGTCA & CCAACCGTGAGAAGATGACT \\
TRX & TGATTTAGGTGGATGAGTTGG & TTCCTCAATATGCCGTACCT \\
Prx3 & GAGCCGAGAAGCAGACGA & ACTCGCACTTCTCCCACC \\
HSP70 & CTGGTTGTCGGAGTAGGTG & ATTTCTCCACAGGCTCCA \\
ATP6L & CCCTCTTCAGTCTTTCCAGTCA & ATTGGTATCGTGGGTGACGC \\
\hline
\end{tabular}


Table 2. Summary of transcriptome characterization of $E$. $j$. sinensis

\begin{tabular}{lcc}
\hline Sequencing & & Control Group \\
\hline Type & Treated Group & 34265370 \\
\hline Raw reads num & 39612948 & 32916952 \\
Clean read num & 38301346 & Transcripts \\
\hline Assembly & & 570847 \\
\hline Type & Unigene & 228410682 \\
\hline Total sequence num & 504889 & 44.59 \\
Total sequence base & 188995620 & 50681 \\
Percent GC & 44.11 & 201 \\
Largest & 50681 & 400.13 \\
Smallest & 201 & 396 \\
Average & 374.33 & 227 \\
N50 & 364 & Transcripts \\
N90 & 224 & 53002 \\
\hline Annotation & & 34443 \\
\hline Type & Unigene & 62284 \\
\hline GO & 42357 & 34761 \\
KEGG & 24773 & 73117 \\
NR & 47038 & 53706 \\
PFAM & 25738 & \\
STRING & 60587 & \\
SWISSPROT & 42963 & \\
\hline & & \\
\hline
\end{tabular}

As an internationally standardized database of predicting the possible functions, COG analysis could help us understand the gene function distribution characteristics of the species. The results showed that of the 504,889 unigenes, 46,552 sequences had a COG classification. From the 25 COG categories, the cluster for "Amino acid transport and metabolism" (4116, 8.84\%) was the largest group, followed by the group for "Replication, recombination and repair" (3993, 8.58\%), "Energy production and conversion" (3482, 7.48\%), "Inorganic ion transport and metabolism" (3095, 6.65\%) and "Carbohydrate transport and metabolism" (3064, 6.58\%) (Figure 2).

Besides COG analysis, 24773 unigenes were successfully mapped to 380 KEGG pathways within 46 categories. Among these pathways, the highly represented categories were "Metabolic pathways" (ko01100: 6637, 26.79\%), "Biosynthesis of secondary metabolites" (ko01110: 2869, 11.58\%), "Biosynthesis of antibiotics" (ko01130: 2215, $8.94 \%$ \%), "Microbial metabolism in diverse environments" (ko01120: 1914, $7.72 \%$ ) and "Carbon metabolism" (ko01200: 1450, 5.85 $\%)$.

\section{Differentially Expressed Genes (DEGs) in the Gill of $E$. $j$. sinensis}

To identify genes display significant changes in expression during Cd stress, we analyzed differentially expressed genes by comparing the $\mathrm{Cd}$ treated library with the control library. The expression level of each unigene was measured by FPKM method. We judged the statistical significance of gene corrected $p$-value $<0.05$ and the $\log _{2}|F C|>=1$. A total of 1544 genes were significant differentially expressed in gills of crabs.
Among these DEGs, 1084 genes were up-regulated and 460 genes were down-regulated compared with the control group (Figure 3 ). This analysis showed $\mathrm{Cd}$ stress had significant effect on the gene expression in $E$. $j$. sinensis, and the larger number of up-regulated genes suggested that most of the DEGs were mainly associated with stress response.

\section{GO Analysis of DEGs}

To obtain a better understanding of biological functions and gene interaction of DEGs, all DEGs were annotated in GO terms and significantly enriched in three GO categories, including Biological Process (BP, 29 sub-categories), Cellular Component (CC, 20 subcategories), and Molecular Function (MF, 15 subcategories) (Figure 4). Within the Biological Process, "cellular process" (GO: 0009987, 275 DEGs), "metabolic process" (GO: 0008152, 239 DEGs), “biological regulation" (GO: 0065007, 211 DEGs), "regulation of biological process" (GO: 0050789, 203 DEGs) and "cellular component organization or biogenesis" (GO: 0071840, 193 DEGs) were mostly prevalent term. Under Cellular Component category, "cell" (GO: 0005623, 285 DEGs) comprised the largest proportion, followed by "cell part" (GO: 0044464, 284DEGs), “organelle" (GO: 0043226, 271 DEGs), “organelle part" (GO: 0044422, 247 DEGs) and "membrane" (GO: 0016020, 222 DEGs). In Molecular Function category, "binding" (GO: 0005488, 229 DEGs) was the predominant GO term, followed by "catalytic activity" (GO: 0003824, 171 DEGs), "transporter activity" (GO: 0005215, 45 DEGs), "molecular function regulator" (GO: 0098772, 39 DEGs) and "structural molecule activity" (GO: 0005198, 28 DEGs). 


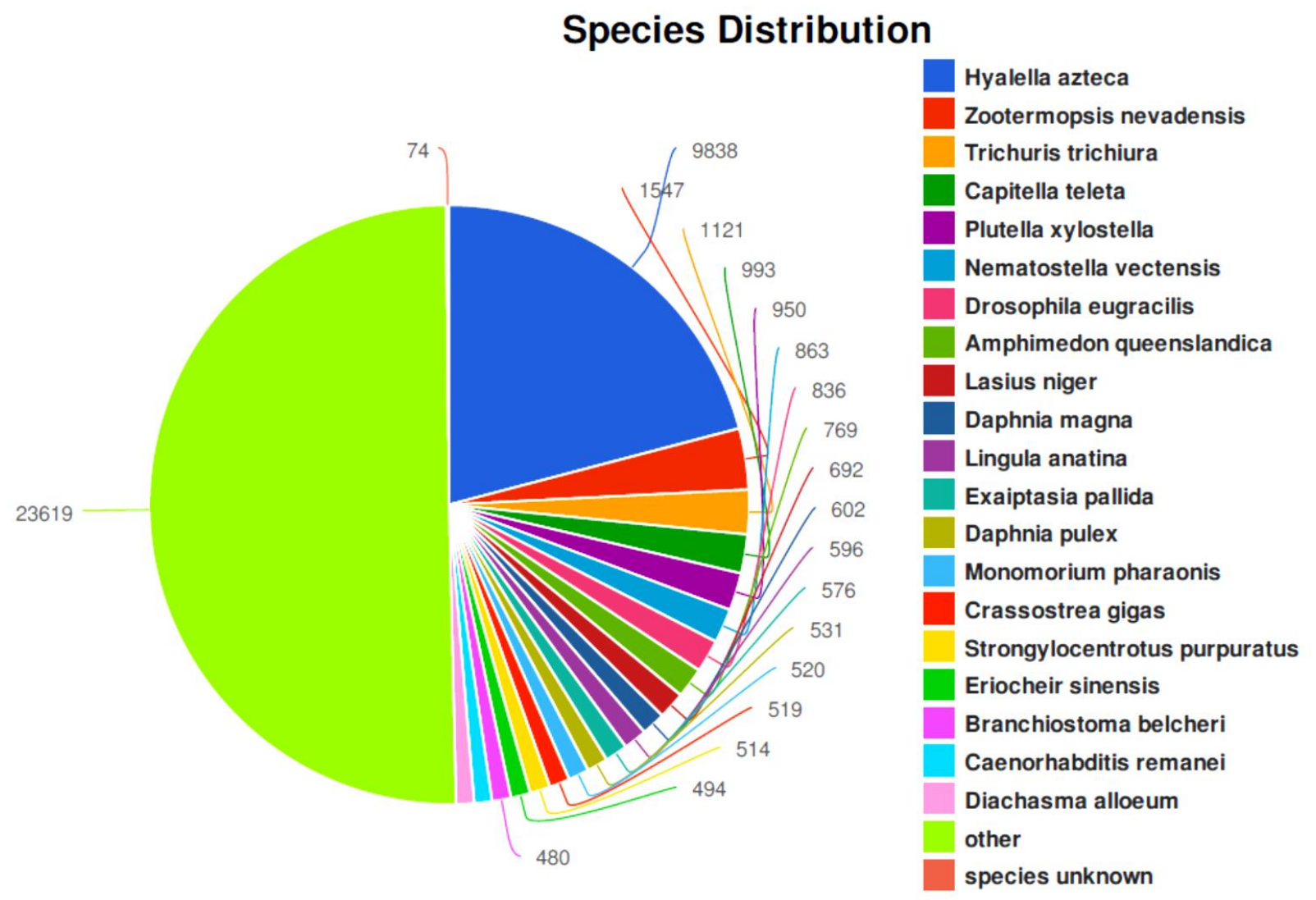

Figure 1. Species distribution of BLASTx matches the hepatopancreas transcriptome unigenes. Each piece of fan indicates the number of top BLAST matches against the Genbank non-redundant ( $\mathrm{Nr}$ ) protein database for various species.

\section{Function Classification}

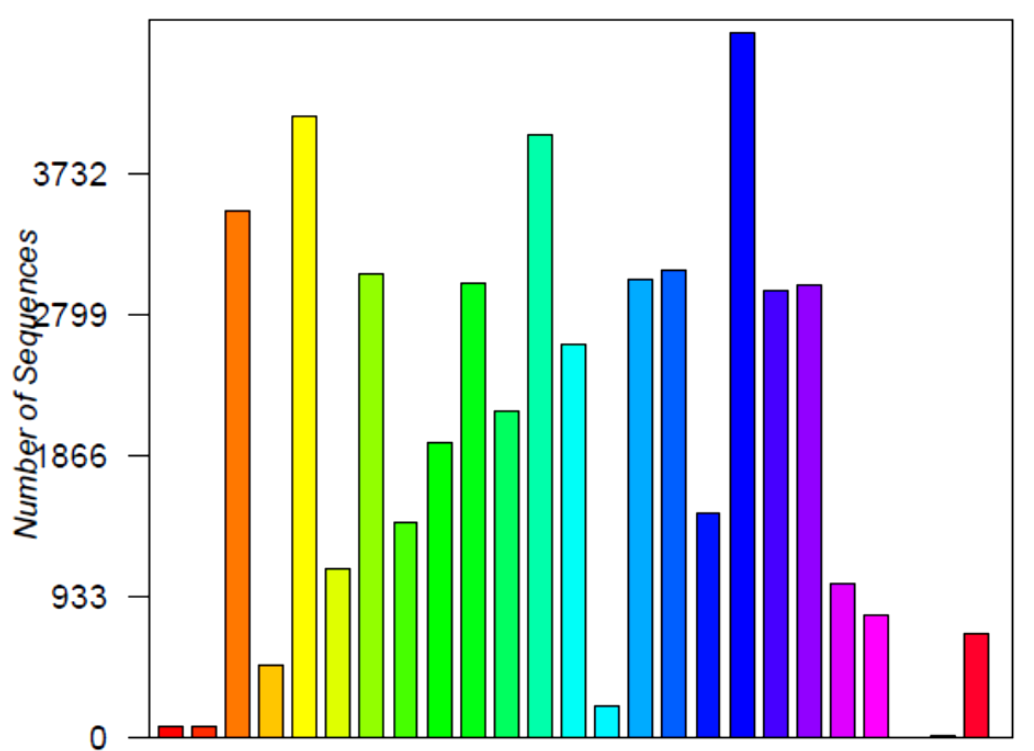

A B C DEFGH I J KLMNOPQRSTUVWYZ

\section{Function Class}

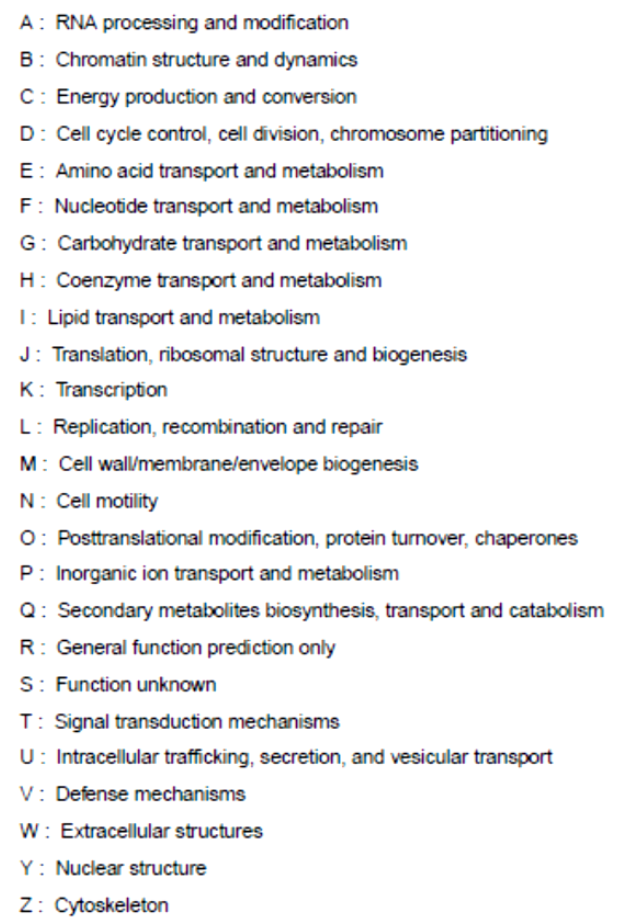

Figure 2. COG classification of all-unigenes. Unigenes were classified into 25 function classes. The columns represents the number of unigenes in each class. 


\section{Cd-treated groups $v S$. controls scatter}

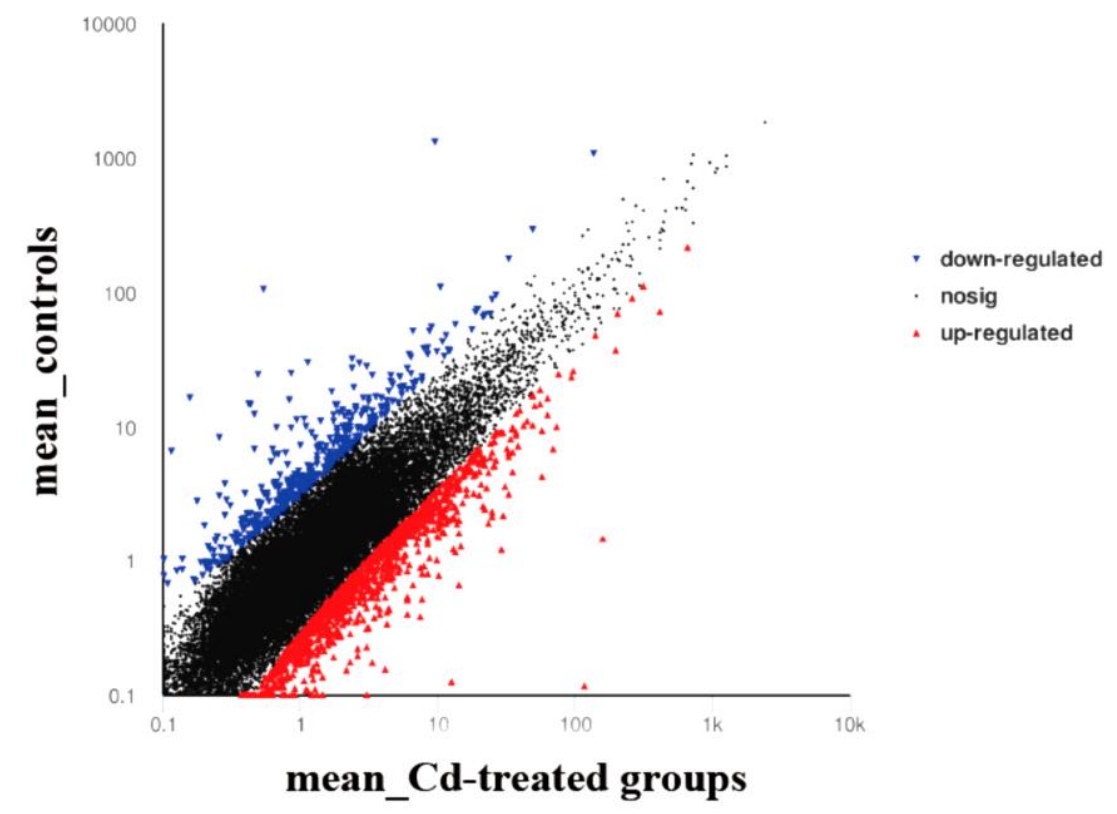

Figure 3. Analysis of DEGs following exposure of the gills to $C d$. The change pattern is displayed as a Scatter plot. The $X$ axis represents the FPKM value [log2(fold change)]. Red points represent upregulated genes and blue points represent downregulated genes based on the discriminative significance values ( fold change $\mid \geq 4.0$ and FDR $\leq 0.001$ ) adopted in this study.

In addition, among all the 64 GO terms, "synapse part", "electron carrier activity", "signal transducer activity", "transcription factor activity, protein binding", "nucleoid", "pigmentation", "presynaptic process involved in chemical synaptic transmission", "cell aggregation", "virion", "virion part", "metallochaperone activity", "detoxification", "translation regulator activity", "carbohydrate utilization", "nitrogen utilization", "protein tag" and "toxin activity" from three GO categories were all up-regulated DEGs with no down-regulated DEGs (Figure 5). It is worth noting that the "synapse part" (14), "electron carrier activity" (11) and "signal transducer activity" (10) categories had the most participating genes in these GO terms. Synapse is a structure that permits a neuron to pass an electrical or chemical signal to another neuron or to the target effector cell. Both "electron carrier activity" and "signal transducer activity" are involved in stress signal transduction (Liu et al., 2017). These findings indicate the importance of signal transduction in the stress response of the $E$. $j$. sinensis caused by Cd stress.

\section{Pathway Analysis of DEGs}

KEGG is a database for biological systems that integrates genomic, chemical, and systemic functional information (Kanehisa et al., 2007). In this study, all DEGs were assigned into KEGG metabolic and regulatory pathways to identify the biological pathways involved in $\mathrm{Cd}$ stress. These genes were mapped into 238 pathways in the KEGG database and 43 pathways were significantly enriched (corrected $P$-value<0.05) (Table 3). The DEGs were mainly concentrated in the pathways of
"Metabolic pathways" (67 up-regulated, 3 downregulated), "Biosynthesis of secondary metabolites" (27 up-regulated, 1 down-regulated), "Oxidative phosphorylation" (26 up-regulated, 0 down-regulated), "Biosynthesis of antibiotics" (23 up-regulated, 0 downregulated) and "Microbial metabolism in diverse environments" (21 up-regulated, 0 down-regulated) (Table 3). These pathways were all classified with metabolites pathway and most DEGs were upregulated. Remarkably, our study found that 29 DEGs were all significant up-regulated in the Oxidative phosphorylation pathway (Figure 4). As we all know, oxidative phosphorylation in mitochondria is a major pathway to supply energy in the basic activities of life through electron transport chain to generate 3'adenosine triphosphate (ATP) (Saraste 1999). DEGs involved in the Oxidative phosphorylation pathway were all significant up-regulated, indicating that $E$. $j$. sinensis needs more ATP to provide more energy for biochemical activities caused by $\mathrm{Cd}$ stress.

Gill associates with ambient environment directly, and it is one of the most important organs involved in osmoregulation in crustaceans (Li et al., 2014). As we all know, $\mathrm{Na}^{+} / \mathrm{K}^{+}$-ATPase (NKA) is a widespread P-type ATPase that plays an important role in the regulation of hematopoietic osmotic pressure (Ahearn et al., 1999; Crambert et al., 2000). In general, alterations to the ion environment could increase membrane fluidity, which may contribute to the changes of NKA enzyme. Here, several pathways directly related to the active regulation of the NKA enzyme were detected, such as "Collecting duct acid secretion" (ko04966), "Dopaminergic synapse" (ko04728) and "Bile secretion" 


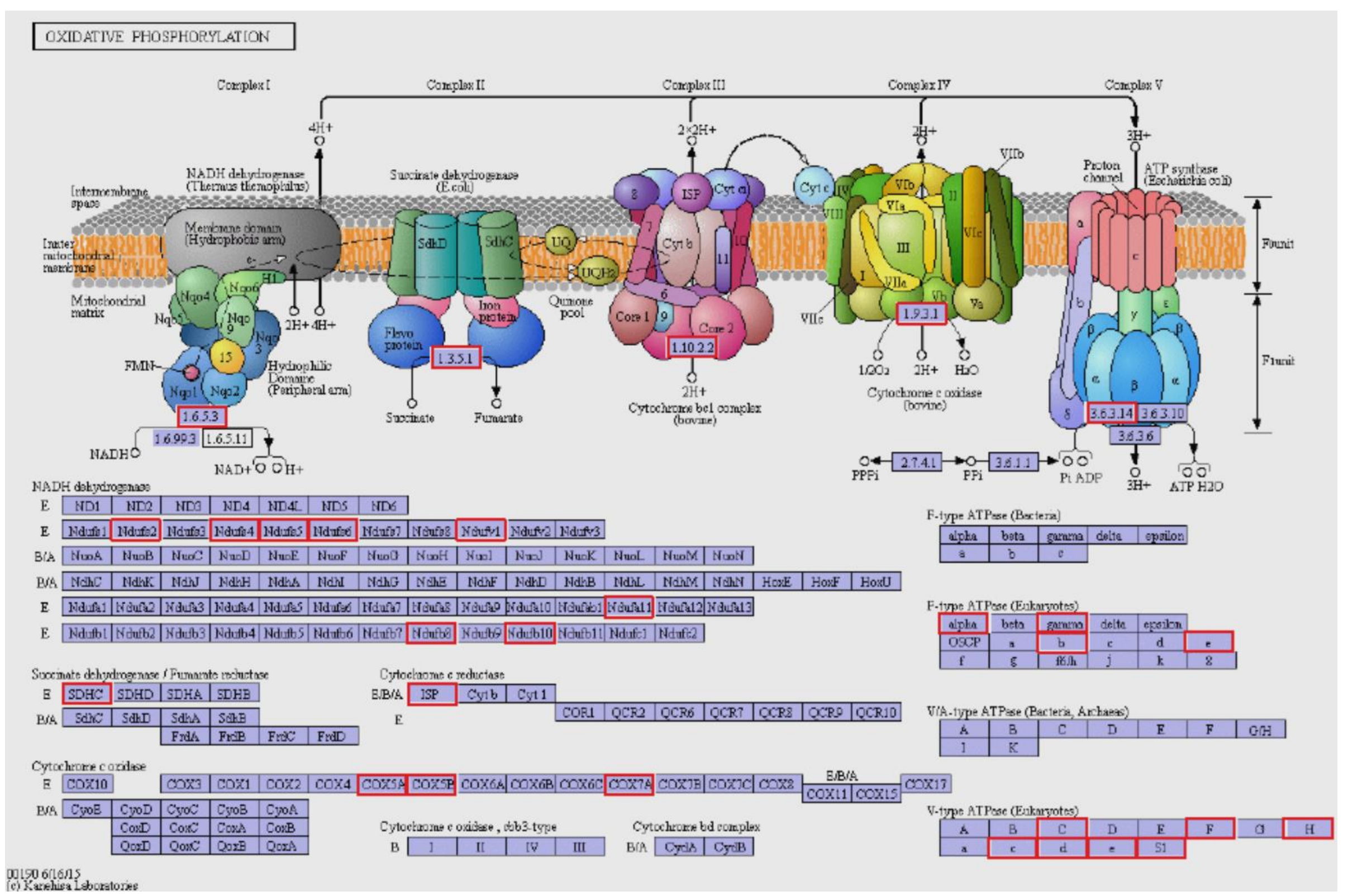

Figure 4. The KEGG pathway of metabolism of Oxidative phosphorylation. The enzymes in the red boxes are associated with the upregulated genes. 
(ko04976) (Table 3). The regulatory genes of NKA in these pathways were all up-regulated, suggesting that the NKA strengthened the ion exchange function necessary to maintain the osmotic balance required for normal survival after $\mathrm{Cd}$ stress. Other regulators were also found in these pathways, including solute carrier organic anion transporter family member 5A1 (SLC5A1), calcineurin B-like protein 1 (CBL1) and serine proteinase inhibitor 2 (SPI2) which may related to metal ion transport $\left(\mathrm{Fe}^{2+}, \mathrm{Cd}^{2+}, \mathrm{Co}^{2+}, \mathrm{Cu}^{2+}, \mathrm{Mn}^{2+}, \mathrm{Ni}^{2+}, \mathrm{Pb}^{2+}, \mathrm{Zn}^{2+}\right)$ and were expressed in the gill (Hui et al., 2017). In addition, three pathways were found that connected with the amino acid metabolism, including "Biosynthesis of amino acids" (ko01230), "Arginine and proline metabolism" (ko00330) and "Arginine biosynthesis" (ko00220) (Table 3). Free amino acids also played a vital role in osmoregulation, the results implied these pathways might all take part in resistant to $\mathrm{Cd}$ stress. In addition, we used cadmium in the form of Cadmium chloride. Chloridion is an essential element for organisms and participate in various kinds of biological function, such as cellular immunologic response, cellular proliferation, differentiation and apoptosis (Xu et al., 2017). Therefore, the active uptake of chloridion also affects various physiological functions of crabs, particularity in osmoregulation. Chinese mitten crab must be required to maintain the proper ion balance, which is need more changes in the adaptive process. Certainly, the mechanism of adaptive process is very complex, and need more in-depth research in the future work.

\section{Identification of Putative Genes for Responding Cd Stress}

We examined the gills transcriptome of Cd-treated and untreated $E$. j. sinensis primarily to identify a variety of candidate genes that might be functionally associated with $\mathrm{Cd}$ toxicity response mechanism. Considering the significance of immune genes in the toxic environment,
$0 \%$

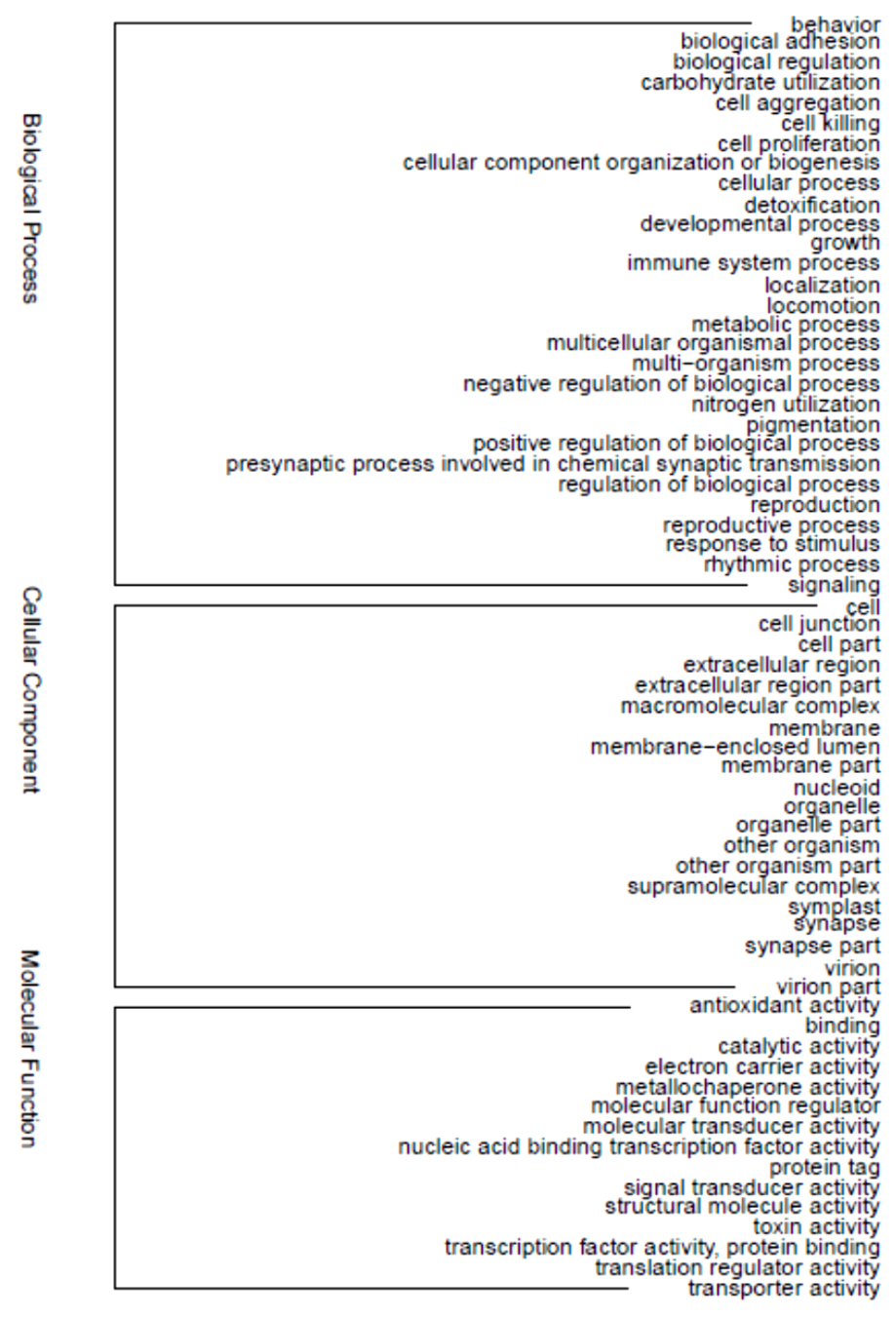

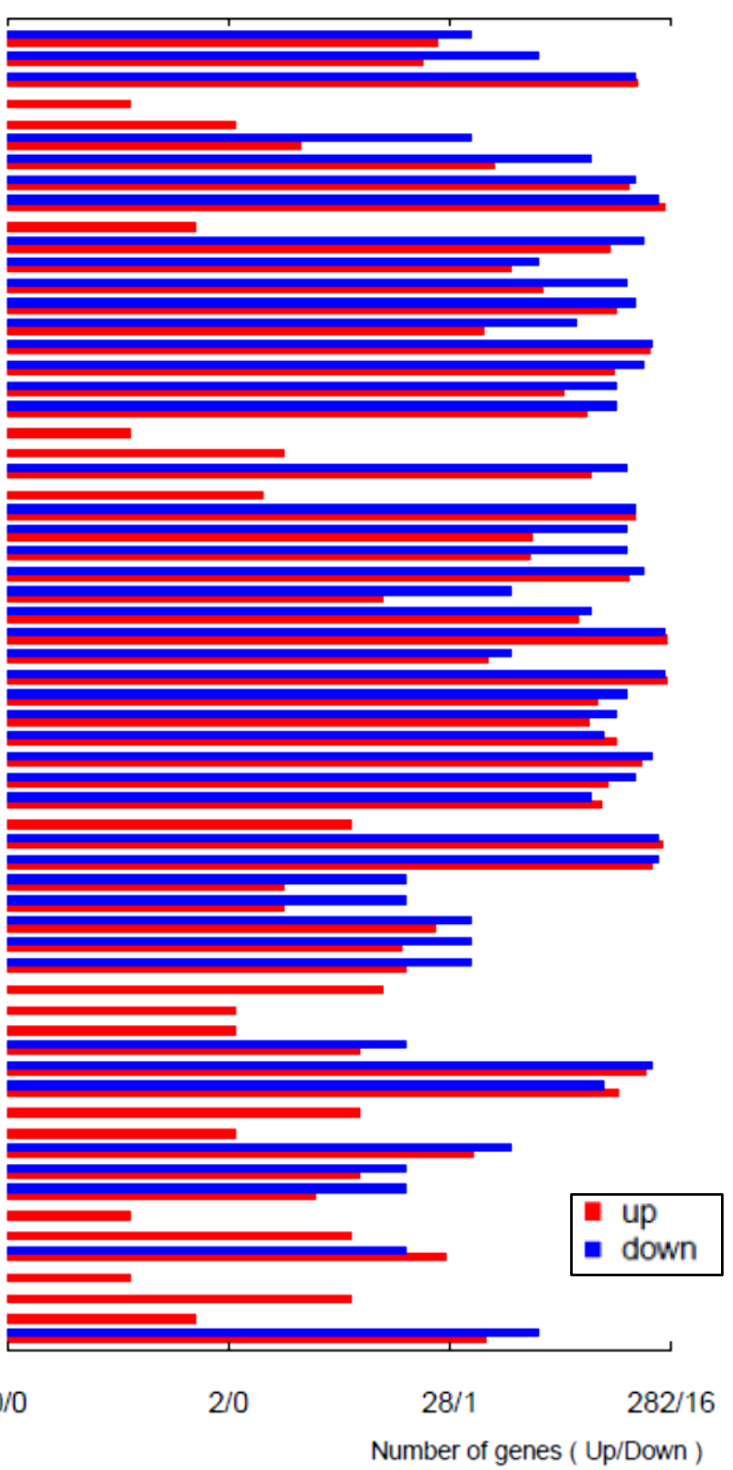

Figure 5. Up- and down-regulated unigenes of the top terms in comparisons between each Cd-treated group and the control. 
Table 3. Significant enrichment of pathways for DEGs between Cd treated crabs and the control. The pathway ID was obtained from the KEGG database. Pathways with a corrected P-value of $<0.05$ were significantly enriched.

\begin{tabular}{|c|c|c|c|c|c|}
\hline $\begin{array}{l}\text { Pathway } \\
\text { ID }\end{array}$ & Pathway & $\begin{array}{l}\text { DEGs associated } \\
\text { with the pathway }\end{array}$ & $\begin{array}{l}\text { Up- } \\
\text { regulated }\end{array}$ & $\begin{array}{l}\text { Down- } \\
\text { regulated }\end{array}$ & $\begin{array}{l}\text { Corrected } \\
\text { P-value }\end{array}$ \\
\hline ko01100 & Metabolic pathways & 70 & 67 & 3 & 0.000072 \\
\hline ko00190 & Oxidative phosphorylation & 29 & 29 & 0 & 0.0000165 \\
\hline ko01110 & Biosynthesis of secondary metabolites & 28 & 27 & 1 & 0.000813172 \\
\hline ko01130 & Biosynthesis of antibiotics & 23 & 23 & 0 & 0.002590833 \\
\hline ko01120 & Microbial metabolism in diverse environments & 21 & 21 & 0 & 0.000336719 \\
\hline ko04141 & Protein processing in endoplasmic reticulum & 21 & 20 & 1 & 0.000997325 \\
\hline ko01200 & Carbon metabolism & 19 & 19 & 0 & 0.000142948 \\
\hline ko05016 & Huntington's disease & 19 & 19 & 0 & 0.006954576 \\
\hline ko05012 & Parkinson's disease & 17 & 17 & 0 & 0.008106575 \\
\hline ko01230 & Biosynthesis of amino acids & 16 & 15 & 1 & 0.0000127 \\
\hline ko05010 & Alzheimer's disease & 16 & 16 & 0 & 0.02056226 \\
\hline ko04932 & Non-alcoholic fatty liver disease (NAFLD) & 14 & 14 & 0 & 0.024560612 \\
\hline ko05418 & Fluid shear stress and atherosclerosis & 12 & 12 & 0 & 0.002404538 \\
\hline ko04145 & Phagosome & 12 & 12 & 0 & 0.002976426 \\
\hline ko05110 & Vibrio cholerae infection & 11 & 11 & 0 & 0.0000426 \\
\hline ko05152 & Tuberculosis & 11 & 11 & 0 & 0.000428232 \\
\hline ko05120 & Epithelial cell signaling in Helicobacter pylori infection & 10 & 10 & 0 & 0.000293024 \\
\hline ko04723 & Retrograde endocannabinoid signaling & 10 & 9 & 1 & 0.038736997 \\
\hline ko05323 & Rheumatoid arthritis & 9 & 9 & 0 & 0.000448045 \\
\hline ko00710 & Carbon fixation in photosynthetic organisms & 8 & 8 & 0 & 0.000370655 \\
\hline ko04721 & Synaptic vesicle cycle & 8 & 8 & 0 & 0.005633931 \\
\hline ko05164 & Influenza A & 8 & 7 & 1 & 0.01375675 \\
\hline ko01212 & Fatty acid metabolism & 7 & 7 & 0 & 0.005633931 \\
\hline ko05134 & Legionellosis & 7 & 5 & 2 & 0.010796747 \\
\hline ko04260 & Cardiac muscle contraction & 7 & 7 & 0 & 0.01375675 \\
\hline ko04966 & Collecting duct acid secretion & 6 & 6 & 0 & 0.004238181 \\
\hline ko00020 & Citrate cycle (TCA cycle) & 6 & 6 & 0 & 0.017624738 \\
\hline ko00071 & Fatty acid degradation & 6 & 6 & 0 & 0.017624738 \\
\hline ko00620 & Pyruvate metabolism & 6 & 6 & 0 & 0.022152894 \\
\hline ko00062 & Fatty acid elongation & 5 & 5 & 0 & 0.004238181 \\
\hline ko00330 & Arginine and proline metabolism & 5 & 4 & 1 & 0.022152894 \\
\hline ko05204 & Chemical carcinogenesis & 5 & 4 & 1 & 0.028603463 \\
\hline ko00030 & Pentose phosphate pathway & 4 & 4 & 0 & 0.010891196 \\
\hline ko01210 & 2-Oxocarboxylic acid metabolism & 4 & 4 & 0 & 0.017720229 \\
\hline ko04976 & Bile secretion & 4 & 4 & 0 & 0.017720229 \\
\hline ko04146 & Peroxisome & 3 & 3 & 0 & 0.006983939 \\
\hline ko00515 & Mannose type O-glycan biosynthesis & 3 & 3 & 0 & 0.006926518 \\
\hline ko00220 & Arginine biosynthesis & 3 & 3 & 0 & 0.028603463 \\
\hline ko00650 & Butanoate metabolism & 3 & 3 & 0 & 0.028603463 \\
\hline ko02020 & Two-component system & 3 & 3 & 0 & 0.045535561 \\
\hline ko00930 & Caprolactam degradation & 2 & 2 & 0 & 0.042557678 \\
\hline ko00960 & Tropane, piperidine and pyridine alkaloid biosynthesis & 2 & 2 & 0 & 0.042557678 \\
\hline ko04614 & Renin-angiotensin system & 2 & 2 & 0 & 0.042557678 \\
\hline
\end{tabular}

we identified 74 up-regulated unigenes and 10 downregulated unigenes in "immune system process", including HSP family members and Proteasome family members. To our knowledge, HSP plays a critical role in molecular chaperones of innate immune responses (Jiang et al., 2009; Wu et al., 2014). Notably, HSP60 play a crucial role in the process of pathogenic and protective immune responses and is implicated in autoimmune disease (Yang et al., 2013). HSP70 could suppress apoptosis by directly binding to Apaf-1, which could prevent recruitment of caspases from the apoptosome complex (Lu et al., 2016). In our research, we noted that many HSP members related to immune resistance had higher levels of expression in treated E. j. sinensis, including HSP60 and HSP70. The increased expression of these HSP genes is in line with previous reports (Sun et al., 2014), which tends to confirm the important role of these proteins in protecting this species from the stress induced by $\mathrm{Cd}$ challenge. Peroxisomes are related to immune responses and antioxidant defence (Blander and Medzhitov 2004; Zhou et al., 2015). Here, proteasome subunit alpha type-4 (PSMA4), proteasome alpha 3 (PSMA3) and proteasome subunit alpha type-5 (PSMA5) were all up-regulated, this increase suggesting the enhancement in immune responses and antioxidant defence of the crab caused by Cd stress

Exposure to heavy metals can promote the generation of ROS in crabs, and ROS production 
promotes oxidation of cellular components and oxidative stress (Zenteno-Savín et al., 2006). Thus, crabs generate some antioxidant enzymes to clear the ROS and avoid oxidative damage. In order to understand the potential molecular mechanism for the damage of $\mathrm{Cd}$ stress, DEGs that associated with "detoxification" and "antioxidant activity" were considered to be potential candidate genes which are important for the response to acute $\mathrm{Cd}$ stress (Table 4). Among the "antioxidant activity" category, 11 DEGs were identified, which comprised 10 up-regulated genes, including glutathione peroxidase (GPX, c398007_g1), glutathione Stransferase (GST, c404726_g2), thioredoxin (TRX, c405622_g2), peroxiredoxin 5 (Prx5, c407190_g1), peroxinectin (Pxt, c407949_g1), peroxiredoxin 3 (Prx3, c412223_g2), prostaglandin D synthase (PGDS, c411308_g2), lipoxygenase (LOX, c411883_g2), glutathione S-transferase (GST, c396511_g1) and protein Smaug homolog 1 (SAM1, c405735_g4). Of which, glutathione S-transferase 1 (GST1, c396511_g1) and protein Smaug homolog 1 (SAM1, c405735_g4) also belonged to "detoxification" category. Among these upregulated genes, GST plays a critical role in protecting organisms against the toxicity of ROS [superoxide anion $\left(\mathrm{O}^{2-}\right)$, hydrogen peroxide $\left(\mathrm{H}_{2} \mathrm{O}_{2}\right)$ and hydroxyl radical $\left(\mathrm{OH}^{-}\right)$] (Roch 1999). GST also is considered as major superfamily of antioxidant enzymes which facilitates the elimination of electrophiles. The increasing of GST activity has also been reported in the swimming crab (Portunus trituberculatus) treated with the elevated ambient ammonia-N (Ren and Pan 2014). Recently, GST has been cloned from the hemolymph of $E$. $j$. sinensis and this research also investigates the distribution of GST mRNA in different tissues in haemocytes of crabs challenged with Aeromonas hydrophila, providing a better understanding of the function of GST gene in this

Table 4. List of identified candidate genes potentially involved in Cd response in $E$. j. sinensis based on gene ontology (GO)

\begin{tabular}{llcc}
\hline Terms from blast hits. & Seq_id & Gene description & Rgulate \\
\hline \multirow{2}{*}{ detoxification } & c396511_g1 & glutathione S-transferase & up \\
toxin activity & c405735_g4 & protein Smaug homolog 1 & up \\
& c407817_g1 & clip domain serine proteinase 2 & up \\
& c398007_g1 & glutathione peroxidase & up \\
& c404726_g2 & glutathione S-transferase & up \\
antioxidant activity & c405622_g2 & thioredoxin & up \\
& c407190_g1 & peroxiredoxin 5 & up \\
& c407949_g1 & peroxinectin & up \\
& c411308_g2 & peroxiredoxin 3 & up \\
\end{tabular}

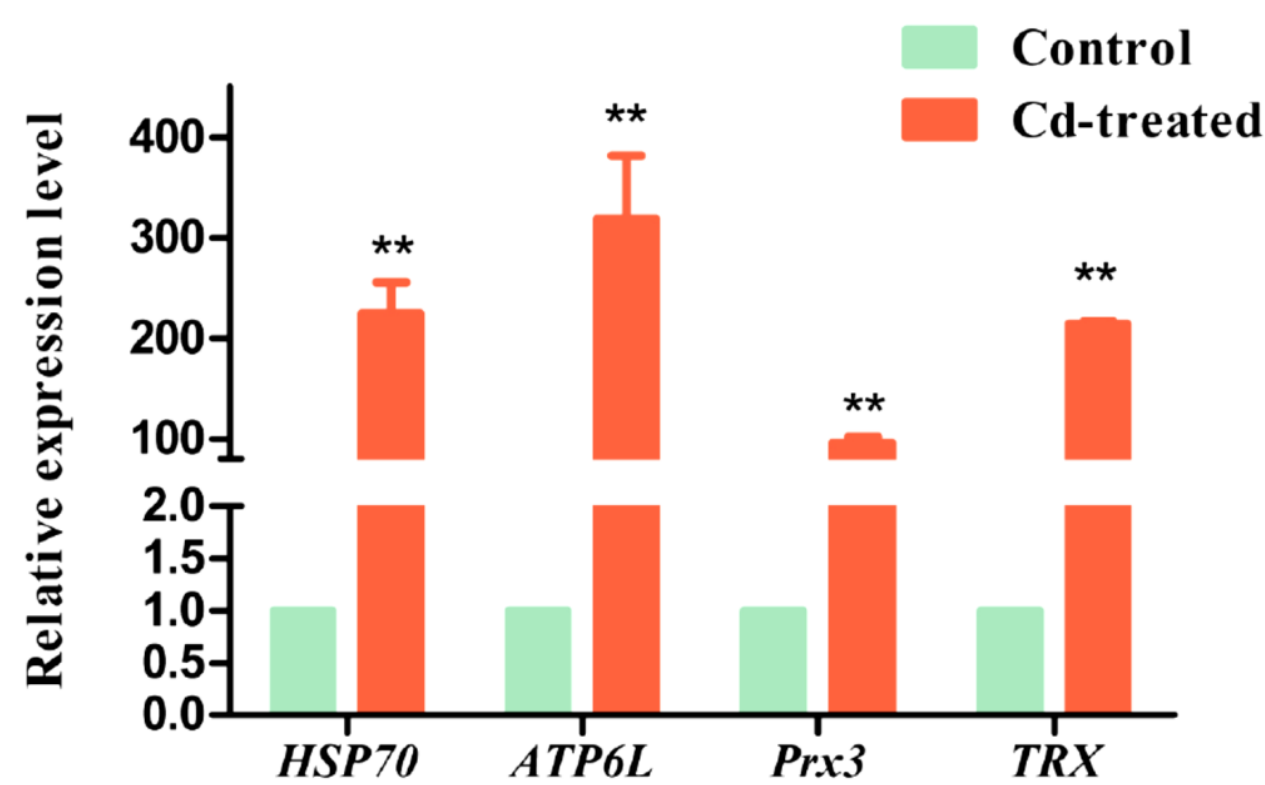

Figure 6. qRT-PCR analysis of the expression level of four Key genes associated with Cd stress. 
species to resist oxidative stress (Zhao et al., 2010). According previous research, GPx is anti-oxidative enzyme that reduces organic and inorganic hydroperoxides to the corresponding alcohols at the expense of reduced glutathione (Borchert et al., 2018). GPX can keep the cellular redox balance and protect against oxidative damage by reducing hydroperoxides at the expense of glutathione (Bain et al., 2016). These results revealed that the upregulation of GST and GPX was involved in stress response and adaptation, and similar upregulation was observed in Sinopotamon henanense (Sun et al. 2016). These genes could be major targets for Cd tolerance, and further experiments are necessary to determine the exact function of these proteins.

\section{Validation of RNA-Seq by qRT-PCR}

To verify the gene expression profile identified by RNA-Seq, 4 genes associated with $\mathrm{Cd}$ response in each comparison were selected for further detection of the relative mRNA expression level by qRT-PCR. The results showed that the expressions of the four genes (HSP70, ATP6L, Prx3, TRX) were more than control group, which were in agreement with the RNA-Seq expression profile (Figure 6). This result also indicates that these genes were involved in the response to $\mathrm{Cd}$.

\section{Conclusion}

In this study, we successfully constructed comparative gills transcriptomes dataset in treated group and control group of Chinese mitten crab and functionally annotated a total of 504,889 unigenes. Most of these genes had an annotation with matches in the NR, String, Swiss-Prot, Pfam, GO and KEGG database. Additionally, we identified 1544 DEGs in the gill after $\mathrm{Cd}$ stress. The results of the GO and KEGG pathway enrichment analyses indicated that the DEGs mainly related to signal transduction, oxidative phosphorylation and osmoregulation were enriched after $\mathrm{Cd}$ stress. A number of genes that are potentially relevant to immune responses, antioxidant, and detoxification were also identified. These results set the stage for expanding the genetic resources available for E. j. sinensis, and also provide important information for further research on the molecular basis of osmoregulation, detoxification and immune response resulting from $\mathrm{Cd}$ stress in crabs.

\section{Acknowledgements}

This study was funded by the National Natural Science Foundation of China (grant number 31702014), and Doctoral Scientific Research Foundation of Yancheng Teachers University to ZFW, and Open Foundation of Jiangsu Key Laboratory for Bioresources of Saline Soils (grant number JKLBS2016007), and Yancheng Teachers University Undergraduate Training
Programs for Innovation and Entrepreneurship to RBL and XLS.

\section{References}

Ahearn, G.A., Duerr, J.M., Zhuang, Z., \& Brown, R.J. (1999). Ion Transport Processes of Crustacean Epithelial Cells. Physiological and Biochenical Zoology, 72(1), 1-18. https://doi.org/10.1086/316643

Altschul, S.F., Madden, T.L., Schäffer, A.A., Zhang, J., Zhang, Z., Miller, W., \& Lipman, D.J. (1997). Gapped BLAST and PSIBLAST: a new generation of protein database search programs. Nucleic Acids Research, 25(17), 3389-3402. https://doi.org/10.1093/nar/25.17.3389

Bain, P.A., Gregg, A.L., \& Kumar, A. (2016). De novo assembly and analysis of changes in the protein-coding transcriptome of the freshwater shrimp Paratya australiensis (Decapoda: Atyidae) in response to acid sulfate drainage water. BMC Genomics, 17-890. https://doi.org/10.1186/s12864-016-3208-y

Blander, J.M., \& Medzhitov, R. (2004). Regulation of Phagosome Maturation by Signals from Toll-like Receptors. Science, 304(5673), 1014-1018. https://doi.org/10.1126/science.1096158

Borchert, A., Kalms, J., Roth, S.R., Rademacher, M., Schmidt, A., Holzhutter, H.G., Kuhn, H., \& Scheerer, P. (2018). Crystal structure and functional characterization of selenocysteine-containing glutathione peroxidase 4 suggests an alternative mechanism of peroxide reduction. Biochimica et Biophysica Acta (BBA) Molecular and Cell Biology of Lipids, 1863(9), 1095-1107. https://doi.org/10.1016/j.bbalip.2018.06.006

Burnett, L.E., \& Mcmahon, B.R. (1985). Facilitation of $\mathrm{CO}_{2}$ excretion by carbonic anhydrase located on the surface of the basal membrane of crab gill epithelium. Respiration Physiology, 62(3), 341-348. https://doi.org/10.1016/0034-5687(85)90089-1

Crambert, G., Hasler, U., Beggah, A.T., Yu, C., Modyanov, N.N., Horisberger, J.D., Lelièvre, L., \& Geering, K. (2000). Transport and pharmacological properties of nine different human $\mathrm{Na}$, K-ATPase isozymes. Journal of Biological Chemistry, 275, 1976-1986. https://doi.org/10.1074/jbc.275.3.1976

Cui, Z., Li, X., Liu, Y., Song, C., Hui, M., Shi, G., Luo, D., \& Li, Y. (2013). Transcriptome profiling analysis on whole bodies of microbial challenged Eriocheir sinensis larvae for immune gene identification and SNP development. Plos One, 8(12), e82156.

https://doi.org/10.1371/journal.pone.0082156

Delanghe, R. (2005). Blast2GO: a universal tool for annotation, visualization and analysis in functional genomics research. Bioinformatics, 21(18), 3674-3676. https://doi.org/10.1093/bioinformatics/bti610

Fu, F.L., \& Qi, W. (2011). Removal of heavy metal ions from wastewaters: a review. Journal of Environmental Management, 92(3), 407-418. https://doi.org/10.1016/j.jenvman.2010.11.011

Gaspic, Z.K., Zvonaric, T., Vrgoc, N., Odzak, N., \& Baric, A. (2002). Cadmium and lead in selected tissues of two commercially important fish species from the Adriatic Sea. Water Research, 36(20), 5023-5028. https://doi.org/10.1016/S0043-1354(02)00111-2

Haas, B.J., Papanicolaou, A, Yassour, M., Grabherr, M., Blood, P.D., Bowden, J., Couger, M.B., Eccles, D., Li, B., \& Lieber, M. (2013). De novo transcript sequence reconstruction 
from RNA-seq using the Trinity platform for reference generation and analysis. Nature Protocols, 8, 1494-1512. https://doi.org/10.1038/nprot.2013.084

Hu, D., Pan, L., Zhao, Q., \& Ren, Q. (2015). Transcriptomic response to low salinity stress in gills of the Pacific white shrimp, Litopenaeus vannamei. Marine Genomics, 24(3), 297-304. https://doi.org/10.1016/j.margen.2015.07.003

Hui, M., Song, C., Liu, Y., Li, C., \& Cui, Z. (2017). Exploring the molecular basis of adaptive evolution in hydrothermal vent crab Austinograea alayseae by transcriptome analysis. Plos One, 12(5), e0178417. https://doi.org/10.1371/journal.pone.0178417

Jiang, S., Qiu, L., Zhou, F., Huang, J., Guo, Y., \& Yang, K. (2009). Molecular cloning and expression analysis of a heat shock protein (Hsp90) gene from black tiger shrimp (Penaeus monodon). Molecular Biology Reports, 36(1), 127-134. https://doi.org/10.1007/s11033-007-9160-9

Kanehisa, M., Araki, M., Goto, S., Hattori, M., Hirakawa, M., Itoh, M., Katayama, T., Kawashima, S., Okuda, S., \& Tokimatsu, T. (2008). KEGG for linking genomes to life and the environment. Nucleic Acids Research, 36(suppl_1), D480-D484. https://doi.org/10.1093/nar/gkm882

Kaoud, H.A., \& Rezk, A. (2011). Effect of exposure to cadmium on the tropical freshwater prawn Macrobrachium rosenbergii. Journal African Journal of Aquatic Science, 36(3), 253-260.

https://doi.org/10.2989/16085914.2011.636899

Li, E., Wang, S., Li, C., Wang, X., Chen, K., \& Chen, L. (2014). Transcriptome sequencing revealed the genes and pathways involved in salinity stress of Chinese mitten crab, Eriocheir sinensis. Physiological genomics, 46(5), 177-190.

https://doi.org/10.1152/physiolgenomics.00191.2013

Li, Q., Wu, X., He, J., Liu, Q., \& Cheng, Y. (2018). Can genetic diversity be maintained during mass selection of the Chinese mitten crab, Eriocheir sinensis?. Aquaculture Research, 49(4), 1606-1615.

https://doi.org/10.1111/are.13616

Liu, Y., Su, H., Li, R., Li, X., Xu, Y., Dai, X., Zhou, Y., \& Wang, H. (2017). Comparative transcriptome analysis of Glyphodes pyloalisWalker (Lepidoptera: Pyralidae) reveals novel insights into heat stress tolerance in insects. BMC Genomics 18, 974. https://doi.org/10.1186/s12864-017-4355-5

Lou, F., Gao, T., Cai, S., \& Han, Z. (2017). De novo assembly and annotation of the whole transcriptome of Oratosquilla oratoria. Marine Genomics. 18, 17-20. https://doi.org/10.1016/j.margen.2017.08.003

Lu, X., Kong, J., Sheng, L., Dai, P., Meng, X., Cao, B., \& Luo, K. (2016). Transcriptome Analysis of the Hepatopancreas in the Pacific White Shrimp (Litopenaeus vannamei) under Acute Ammonia Stress. Plos One,11(10), e0164396. https://doi.org/ 10.1371/journal.pone.0164396

Manna, P., \& Sil, P.C. (2009). Taurine plays a beneficial role against cadmium-induced oxidative renal dysfunction. Amino Acids, 36, 417-428. https://doi.org/10.1007/s00726-008-0094-x

Mortazavi, A., Williams, B.A., Mccue, K., Schaeffer, L., \& Wold, B. (2008). Mapping and quantifying mammalian transcriptomes by RNA-Seq. Nature Methods, 5(7), 621628. https://doi.org/10.1038/nmeth.1226

Overstreet, J.D. (2000). Effects of arsenic, cadmium, chromium, and lead on gene expression regulated by a battery of 13 different promoters in recombinant HepG2 cells. Toxicology and Applied Pharmacology, 168(2), 7990. https://doi.org/10.1006/taap.2000.9014

Ren, Q., \& Pan, L. (2014). Digital gene expression analysis in the gills of the swimming crab (Portunus trituberculatus) exposed to elevated ambient ammonia-N. Aquaculture, 434(20), 108-114.

https://doi.org/10.1016/j.aquaculture.2014.08.008

Roch, P. (1999). Defense mechanisms and disease prevention in farmed marine invertebrates. Aquaculture, 172(1-2), 125-145. https://doi.org/10.1016/S0044-8486(98)00439-6

Saraste, M. (1999). Oxidative Phosphorylation at the fin de siècle. Science, 283, 1488-1493. https://doi.org/10.1126/science.282.5407.1488

Silvestre, F., Dierick, J.F., Dumont, V., Dieu, M., Raes, M., \& Devos, P. (2006). Differential protein expression profiles in anterior gills of Eriocheir sinensis during acclimation to cadmium. Aquatic toxicology, 76(1), 46-58. https://doi.org/10.1016/j.aquatox.2005.09.006

Silvestre, F., Duchêne, C., Trausch, G., \& Devos, P. (2005). Tissue-specific cadmium accumulation and metallothionein-like protein levels during acclimation process in the Chinese crab Eriocheir sinensis. Comparative Biochemistry Physiology Part C:Toxicology \& Pharmacology, 140(1), 39-45. https://doi.org/10.1016/j.cca.2005.01.004

Sun, M., Li, Y.T., Liu, Y., Shao, C.L., \& Wang, L. (2016). Transcriptome assembly and expression profiling of molecular responses to cadmium toxicity in hepatopancreas of the freshwater crab Sinopotamon henanense. Scientific report, 6, 19405. https://doi.org/10.1038/srep19405

Sun, S., Xuan, F., Ge, X., Fu, H., Zhu, J., \& Zhang, S. (2014). Identification of differentially expressed genes in hepatopancreas of oriental river prawn, Macrobrachium nipponense exposed to environmental hypoxia. Gene, 534(2), 298-306. https://doi.org/10.1016/j.gene.2013.10.036

Thévenod, F. (2009). Cadmium and cellular signaling cascades: to be or not to be? Toxicology and applied pharmacology, 238(3), 221-239.

https://doi.org/10.1016/j.taap.2009.01.013

Thomas, S., Kenneth, D., \& Livak, J. (2008). Analyzing real-time PCR data by the comparative CT method. Nature Protocols, 3(6), 1101-1108. https://doi.org/10.1038/nprot.2008.73

Wang, J., Ren, T., Han, Y., Zhao, Y., Liao, M., Wang, F., \& Jiang, Z. (2015). Effects of dietary vitamin $C$ supplementation on lead-treated sea cucumbers, Apostichopus japonicus. Ecotoxicology and Environmental Safety, 118, 21-26. https://doi.org/10.1016/j.ecoenv.2015.04.009

Wang, J., Zhang, P., Shen, Q., Wang, Q., Liu, D., Li, J., \& Wang, L. (2013). The effects of cadmium exposure on the oxidative state and cell death in the gill of freshwater crab Sinopotamon henanense. Plos One, 8(5), e64020. https://doi.org/10.1371/journal.pone.0064020

Wang, L., Yang, X., Wang, Q., \& Wang, D. (2001). The accumulation of $\mathrm{Cd}^{\sim}(2+)$ and the effect on est in five tissues and organs of Eriocheir sinensis. Acta Zoologica Sinica, 47(Suppl.), 96-100. https://doi.org/1522515

Wang, Z., Bai, Y., Zhang, D., \& Tang, B. (2018). Adaptive evolution of osmoregulatory-related genes provides insight into salinity adaptation in Chinese mitten crab, Eriocheir sinensis. Genetica, 146(3), 1-9. https://doi.org/10.1007/s10709-018-0021-3 
Weihrauch, D., Ziegler, A., Siebers, D., \& Towle, D.W. (2001). Molecular characterization of V-type H(+)-ATPase (Bsubunit) in gills of euryhaline crabs and its physiological role in osmoregulatory ion uptake. Journal of Experimental Biology, 204(1), 25-37.

https://doi.org/11104708

Wu, X., Cheng, Y., Sui, L., Zeng, C., Southgate, P.C., \& Yang, X. (2007). Effect of dietary supplementation of phospholipids and highly unsaturated fatty acids on reproductive performance and offspring quality of Chinese mitten crab, Eriocheir sinensis ( $H$. MilneEdwards), female broodstock. Aquaculture, 273(4), 602613. https://doi.org/10.1016/j.aquaculture.2007.09.030

Wu, X., Tan, J., Cai, M., \& Liu, X. (2014). Molecular cloning, characterization, and expression analysis of a heat shock protein (HSP) 70 gene from Paphia undulata. Gene, 543(2), 275-285.

https://doi.org/10.1016/j.gene.2013.11.103

Xiong, L.W., Wang, Q., \& Qiu, G.F. (2001). Resources and Culturing Situation of Chinese Mitten Crab (Eriocheir sinensis) and Species Character Conservation. Journal of Lake Sciences, 13(3), 267-271. https://doi.org/10.18307/20010311

Xu, B. P., Tu, D. D., Yan, M. C., Shu, M. A., \& Shao, Q. J. (2017). Molecular characterization of a cDNA encoding $\mathrm{Na}+/ \mathrm{K}+/ 2 \mathrm{Cl}$ - cotransporter in the gill of mud crab (Scylla paramamosain) during the molt cycle: Implication of its function in osmoregulation. Comp Biochem Physiol A Mol Integr Physiol, 203, 115-125. https://doi.org/ 10.1016/j.cbpa.2016.08.019

Yang, Y., Ye, H., Huang, H., Li, S., Zeng, X., Gong, J., \& Huang, X. (2013). Characterization and expression of SpHsp60 in hemocytes after challenge to bacterial, osmotic and thermal stress from the mud crab Scylla paramamosain. Fish \& Shellfish Immunology, 35(4), 1185-1191. https://doi.org/10.1016/j.fsi.2013.07.029

Zenteno-Savín, T., Saldierna, R., \& Ahuejote-Sandoval, M. (2006). Superoxide radical production in response to environmental hypoxia in cultured shrimp. Comparative Biochemistry and Physiology Part C: Toxicology \& Pharmacology, 142(3-4), 301-308.

https://doi.org/10.1016/j.cbpc.2005.11.001

Zhao, D., Chen, L., Qin, C., Zhang, H., Wu, P., \& Zhang, F. (2010). A delta-class glutathione transferase from the Chinese mitten crab Eriocheir sinensis: cDNA cloning, characterization and mRNA expression. Fish \& Shellfish Immunology, 29(4), 698-703. https://doi.org/10.1016/j.fsi.2010.06.002

Zhou, M.T., Qin, Y., Li, M., Chen, C., Chen, X., Shu, H.B., \& Guo, L. (2015). Quantitative Proteomics Reveals the Roles of Peroxisome-associated Proteins in Antiviral Innate Immune Responses. Molecular \& Cellular Proteomics, 14(9), 25. https://doi.org/10.1074/mcp.m115.048413 